\title{
gs \\ Two-dimensional magnetic resonance force microscopy using full-volume Fourier and Hadamard encoding
}

\author{
Kai W. Eberhardt, ${ }^{1}$ Andreas Hunkeler, ${ }^{1}$ Urban Meier, ${ }^{1}$ Joy Tharian, ${ }^{2}$ Schahrazede Mouaziz, ${ }^{3}$ Giovanni Boero, ${ }^{3}$ \\ Jürgen Brugger, ${ }^{3}$ and Beat H. Meier ${ }^{1, *}$ \\ ${ }^{1}$ Physical Chemistry, ETH Zurich, CH-8093 Zurich, Switzerland \\ ${ }^{2}$ EMPA Materials Science and Technology, Ueberlandstrasse 129, 8600 Duebendorf, Switzerland \\ ${ }^{3}$ Microsystems Laboratory, Ecole Polytechnique Fédérale de Lausanne (EPFL), Lausanne 1015, Switzerland \\ (Received 28 July 2008; revised manuscript received 16 October 2008; published 1 December 2008)
}

\begin{abstract}
A rapid acquisition method for two-dimensional (2D) magnetic resonance force microscopy images is described. Spatial resolution is based on gradients in the static magnetic field $B_{0}$ and in the applied radiofrequency field $B_{1}$. Hadamard- and Fourier-encoding schemes, respectively, are employed to obtain high sensitivity by taking advantage of a maximum multiplex effect. $2 \mathrm{D}$ images of a patterned $\left(\mathrm{NH}_{4}\right)_{2} \mathrm{SO}_{4}$ crystal sample are reconstructed from the known field distributions with a resolution of $1 \mu \mathrm{m}$.
\end{abstract}

DOI: 10.1103/PhysRevB.78.214401

PACS number(s): 76.60.Pc

\section{INTRODUCTION}

Magnetic resonance imaging (MRI) is a well-established noninvasive method to image a wide range of objects from materials to humans. It is a particular strength of MRI that a multitude of object properties can be mapped onto the spatial image by choosing an appropriate image contrast method. MRI microscopy is presently limited to a resolution of a few micrometers if mobile water is imaged ${ }^{1,2}$ and generally worse for materials having broader NMR linewidth, in particular solids., 3 These limitations are a consequence of the relatively low NMR detection sensitivity and the limited size of the achievable magnetic-field gradient. Further improvement, e.g., to obtain the resolution of optical microscopes, demands for alternative approaches. Magnetic resonance force microscopy (MRFM) is a promising approach that addresses both issues mentioned. While the MRI experiment detects magnetization inductively, using one or several radiofrequency (rf) coils, the MRFM experiments described here detect the mechanical oscillation amplitude of a micromechanical cantilever. The oscillation is driven by the force acting on the sample when placed in a large magnetic-field gradient $^{5-9}$ and in the presence of a rf field which inverts the magnetization in a preselected slice of the sample. Through this process, the force on the nuclear magnetization in the active slice is modulated at the mechanical resonance frequency of a high- $Q$ cantilever with optimized force sensitivity and the mechanical oscillation is detected. It has been demonstrated that for small samples and detectors (micrometers or less), the mechanical detection becomes more sensitive than inductive detection. ${ }^{10}$ Large field gradients needed for sensitive detection and high spatial resolution are easily produced by positioning a small ferromagnet in close proximity to the sample.

Three-dimensional (3D) imaging with MRFM has been demonstrated using a scanning-probe technique where the tip is moved over the sample in two dimensions (2D). At each position, a signal is recorded which is proportional to the number of spins in the sensitive volume. The shape of this volume is defined by the geometry of the ferromagnetic gradient tip, typically a slice curved to a half sphere. The thick- ness of the slice is defined by the excitation bandwidth of the rf irradiation. The signal from different depths in the sample - the third dimension-is obtained by varying the frequency of the rf irradiation which defines the distance of the sensitive slice to the gradient source. The image is reconstructed by deconvolving the measured 3D data set with the profile of the magnetic-field gradient. First images were presented by Züger et al., ${ }^{11,12}$ obtaining a resolution of $3.6 \mu \mathrm{m}$ in a $3 \mathrm{D}$ image of $\left(\mathrm{NH}_{4}\right)_{2} \mathrm{SO}_{4}$ and similar experiments are reported in Refs. 13 and 14. Recently Mamin et al. ${ }^{15} \mathrm{dem}-$ onstrated $90 \mathrm{~nm}$ spatial resolution at cryogenic temperatures.

NMR imaging methods are generally based on the idea that the spatial image is encoded in the spin-resonance frequencies detected in the presence of a magnetic-field gradient: higher magnetic fields lead to higher Larmor or Rabi precession frequencies. 3D images can be obtained by sequentially switching on static field gradients in three orthogonal spatial directions. In rotating-frame zeugmatography, first demonstrated by Hoult, ${ }^{16}$ gradients in the strength of the rf field $B_{1}$ can be used as an alternative to gradients in the static field $B_{0}$. For clinical MRI, they are seldom used but in MRI microscopy this method can be advantageous. ${ }^{17}$ In general, MRI uses Fourier encoding to obtain spatial resolution, allowing for a simultaneous acquisition of information from the entire sample and leaving the relative position of sample and detector constant. ${ }^{18,19}$ This scheme cannot easily be adapted to MRFM as it requires quickly switchable field gradients. Nevertheless, the idea of multiplexed acquisition can also be implemented with constant field gradients using Hadamard-encoding techniques. ${ }^{20}$

Here we present a two-dimensional spatial encoding scheme for MRFM which uses a combination of Hadamard and Fourier encoding in the direction of the static field $B_{0}$ and the rf field $B_{1}$, respectively. Each acquisition collects data from the entire three-dimensional sample volume, offering the full multiplex advantage. The method can also be extended to three-dimensional imaging.

\section{RESULTS}

To assess the fidelity and the resolution of our 2D imaging scheme, a test sample in the form of the three letters ETH 


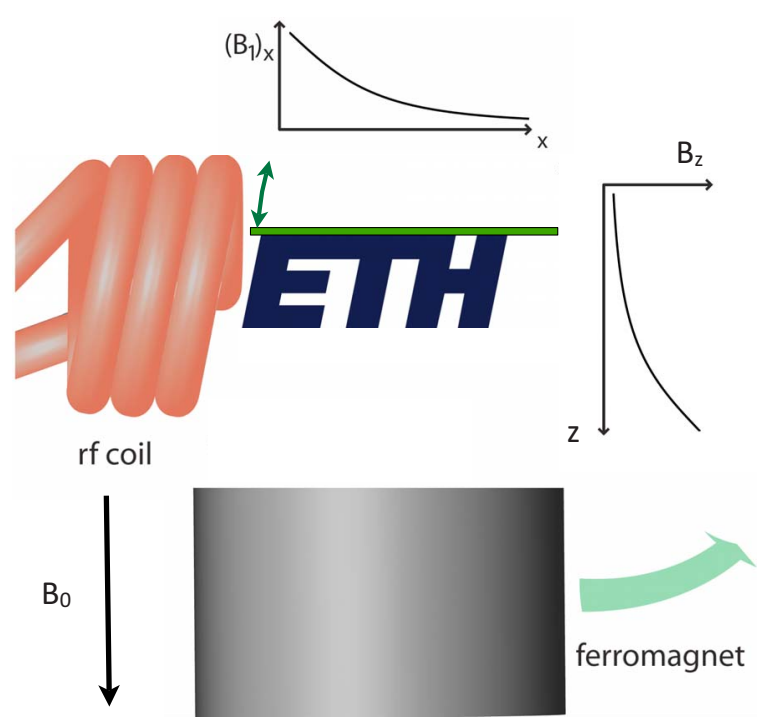

FIG. 1. (Color online) Rotating-frame MRFM setup. To create an image of the sample, here a $\left(\mathrm{NH}_{4}\right)_{2} \mathrm{SO}_{4}$ crystal glued to the cantilever (indicated by bar and vibrational direction by a double arrow), we encode the magnetization in two dimensions over the sample. In the $z$ direction we encode the spatial position of the magnetization by the Larmor frequency of the spins in the inhomogeneous field produced by an iron cylinder gradient source. In the $x$ direction we use an rf pulse, which nutates the spins closer to the coil entrance faster than the spins further away due to the gradient in the $B_{1}$ field, creating a Fourier encoding over the object. As the field gradient produced by the iron cylinder would influence the nutation frequencies by off-resonance contributions, we flip away the gradient source with a piezo bender for the duration of the $x$-encoding pulse.

with an overall size of $28 \times 8 \mu \mathrm{m}^{2}$ and a maximum thickness of $10 \mu \mathrm{m}$ was produced from a single crystal of $\left(\mathrm{NH}_{4}\right)_{2} \mathrm{SO}_{4}$ by a focused ion beam (FIB). The sample was mounted in the force microscope as indicated in Fig. 1. Note that the sample is shown much larger than to scale in the figure. The field gradients along two orthogonal directions $z$ and $x$ (see Fig. 1 for coordinate system orientation) were calculated from the formula given in Secs. III A and III B. Along the $z$ direction a gradient in the $B_{0}$ field is generated by a ferromagnetic cylinder; along the $x$ direction, a $B_{1}$-field gradient is produced by the $\mathrm{rf}$ coil. These two gradients are employed for imaging using the pulse sequence of Fig. 2. During the $x$-encoding period, the magnetization nutates around the rf-field direction ( $x$ axes) with a Rabi nutation angle proportional to the product of $B_{1}$-field strength and pulse duration. In our experimental implementation, we systematically vary the pulse length while keeping the amplitude constant. Due to the presence of the field gradient along $x$, the nutation frequency encodes the $x$ position. During this time period, the gradient source is mechanically flipped away thereby greatly reducing the $B_{0}$-field gradient to avoid offresonance effects on the nutation. After the $x$ encoding, the (unwanted) transverse magnetization component decays during a $200 \mathrm{~ms}$ delay while the gradient source is flipped back into the position indicated in Fig. 1; whereas the $z$ component survives and is used to form the image. For simultaneous signal acquisition over the entire sample volume, the

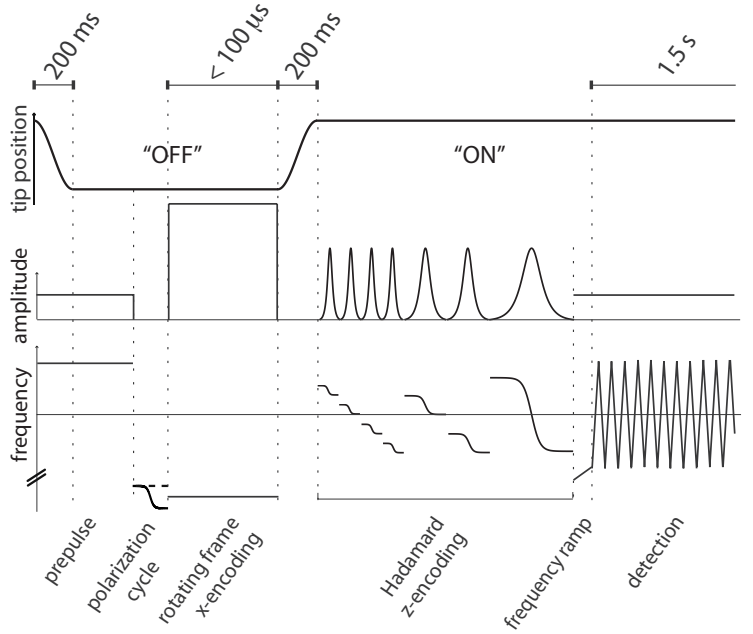

FIG. 2. Pulse sequence for 2D MRFM rotating-frame imaging. The gradient source is flipped away with a piezo bender actuator during the first off-resonance pulse, which does not act on the spins but preheats the cantilever and reduces artifacts. The following rf pulse performs the rotating-frame $x$ encoding. The Rabi nutation frequency is about $\frac{\omega_{1}}{2 \pi} \approx 350 \mathrm{kHz}$ at the sample center. The rf field is set to relatively high values to minimize offset effects in the residual $B_{0}$ inhomogeneity and to minimize the encoding time required. After the gradient is flipped back the magnetization is encoded in the $z$ direction by a Hadamard-16 sequence and finally detected.

resonance frequencies of the individual slices are encoded by a Hadamard scheme implemented by frequency selective inversion pulses. ${ }^{20} \mathrm{~A}$ set of slices is inverted by a series of frequency selective pulses and by acquiring the signal from the region of the collected slices. By linear combination of the thus measured data the intensity of the individual slices can be reconstructed with a higher signal-to-noise (SNR) ratio than if the slices were acquired successively. Then, the remaining longitudinal magnetization is detected under the static field gradient in $z$ direction by cyclic adiabatic inversion of the spins. The $y$ direction is not encoded in our current setup and the image is a projection of the spin density onto the reconstructed 2D plane.

Due to the spatial variation of the field gradients, as shown in Fig. 4, the resolution over the object is not homogeneous in $x$ or $z$. The calculated field profiles were taken into account for image reconstruction, as described in Secs. III $\mathrm{A}$ and III $\mathrm{B}$.

The MRFM image of the test sample shown in Fig. 3(a) compares favorably with the scanning electron microscopy (SEM) image of the sample [Figs. 3(b) and 3(c)]. Note that for production-technical reasons, the sample is not of uniform thickness and because the pixel intensity in the MRFM image represents the integral intensity of the entire thickness of the sample, the intensity over the letters is not uniform. As expected from the SEM pictures [Figs. 3(b) and 3(c)], the MRFM signal intensity decreases toward the bottom of the logo and toward the left side of the letter E. The mean resolution given by our encoding is 0.8 and $1 \mu \mathrm{m}$ in $z$ and $x$ directions, respectively. The width of the sample ranges from 1 to $10 \mu \mathrm{m}$; therefore we have imaged voxels between ap- 

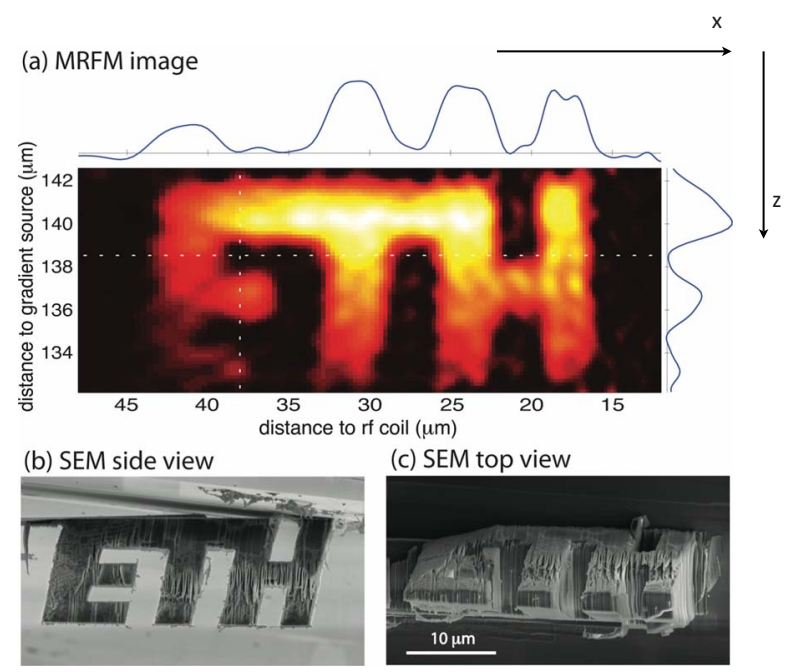

FIG. 3. (Color online) (a) 2D MRFM image of the test sample. The signal intensity at the position of the dotted lines is plotted on top and to the right of the image. (b) SEM micrograph of the same sample. The view is approximately the same as for (a). (c) Top view of the sample. The logo is clearly thinner at the bottom and at the beginning of the E: a feature reproduced by the signal intensity of image (a).

proximately 1 and $10 \mu \mathrm{m}^{3}$ in volume. The smallest voxels correspond to $6 \times 10^{10}$ spins, with a net Boltzmann polarization corresponding to $2 \times 10^{5}$ fully polarized spins.

The MRFM image reproduces all the features of the sample and no artifacts are visible, only the slant of the "italic" letters is not perfectly reproduced, probably because the $B_{1}$ - and $B_{0}$-field gradients are not perfectly orthogonal in our setup.

\section{METHODS}

\section{A. Setup}

The MRFM probe used has been described before. $23,30,31$ It operates under vacuum conditions $\left(10^{-6} \mathrm{mbar}\right)$ at room temperature in a $6.0 \mathrm{~T}$ wide-bore NMR magnet. The cantilever is oriented orthogonal to the field gradient and operated in amplitude mode..$^{7,32,33}$ In addition, a piezo bender actuator (Physikinstrumente, Germany) was used to flip the gradient source $2 \mathrm{~mm}$ away from the sample to reduce the $B_{0}$ gradient during encoding with $B_{1}$. To minimize mechanical disturbance by the flip of the gradient source we ramp the voltage that controls the flipper piezo according to a sine function (indicated in Fig. 2) within $100 \mathrm{~ms}$ and wait for another 100 ms. Under the action of the active dampening mechanism, the ring-down time of the cantilever is in the order of $10 \mathrm{~ms}$ and by waiting more than $100 \mathrm{~ms}$ the residual motion is well below the noise level. As the gradient source for the $B_{0}$ field we used an iron cylinder of $100 \mu \mathrm{m}$ radius. The field from the cylindrical gradient source $(L=10 \mathrm{~mm}, R=100 \mu \mathrm{m}$, and $\left.B_{\text {sat }}=1.75 \mathrm{~T}\right)$ on the symmetry axes of the cylinder is

$$
B_{0, z}(z)=\frac{B_{\mathrm{sat}}}{2}\left[\frac{z+L}{\sqrt{(z+L)^{2}+R^{2}}}-\frac{z}{\sqrt{z^{2}+R^{2}}}\right] .
$$

To generate the $B_{1}$ field, a $50 \mu \mathrm{m}$ inner diameter solenoid coil with four windings from a 50 - $\mu \mathrm{m}$-isolated copper wire was employed. The $B_{1}$-field amplitude from the coil is approximately orthogonal to the $B_{0}$ field and falls off, on the $x$ axis, with the distance $x$ to the coil as

$$
B_{1, x}(x)=\frac{\mu_{0} I N_{I}}{2}\left[\frac{x+L}{\sqrt{(x+L)^{2}+R^{2}}}-\frac{x}{\sqrt{x^{2}+R^{2}}}\right],
$$

with the current through the coil $I$ and the windings per length of coil $N_{I}$. The above equations are for the field on the axes of the static gradient source and the solenoid coil, respectively, and are a good approximation over the entire diameter of the sample, which is much smaller than the coil and cylinder diameters.

The single crystal $\left(\mathrm{NH}_{4}\right)_{2} \mathrm{SO}_{4}$ sample was glued to the tip of a custom-made silicon cantilever ${ }^{21} \quad\left(k=130 \frac{\mu \mathrm{N}}{m}, f_{c}\right.$ $=833 \mathrm{~Hz}$, and $Q \approx 3 k$ ) and then cut to shape the letters ETH on an area of $28 \times 8 \mu \mathrm{m}^{2}$ by a FIB.

\section{B. Acquistion and image reconstruction}

The 2D image was resolved by recording 70 and 16 image points in the $x$ and $z$ directions, respectively. This scan was repeated eight times for signal averaging, leading to a total recording time of $42 \mathrm{~h}$. The $B_{1}$-field strength for $x$ encoding ranged from 180 to $520 \mathrm{kHz}$ depending on the position in the sample. During signal acquisition the mean value, over the sample, of the rf field used for the cyclic adiabatic inversions was set to $66 \mathrm{kHz}$. The acquisition time was $1.5 \mathrm{~s}$.

Because the $B_{0, z}$ and $B_{1, x}$ field gradients are orthogonal within the limits of alignment, two dimensions of the image can be reconstructed independently. For the $z$ direction, along the $B_{0}$ gradient, Hadamard-16 encoding was used and the information was reconstructed accordingly. ${ }^{20}$ To transform the Larmor frequencies to spatial positions along the $z$ axis we calculated the field profile from a cylindrical gradient source $\left(L=10 \mathrm{~mm}, R=100 \mu \mathrm{m}\right.$, and $\left.B_{\text {sat }}=1.75 \mathrm{~T}\right) \mathrm{ac}$ cording to Eq. (1) from which we can assign a spatial $z$ coordinate to the Larmor precession frequency of each slices of the Hadamard reconstruction. The 16-point image obtained was trigonometrically interpolated to 64 points.

For reconstruction of the $x$ direction (along the $B_{1}$ gradient) the $70 \mathrm{k}$-space (inverse space) data points obtained by incrementing the length of the nutation pulse were zero filled to obtain 512 points and subsequently Fourier transformed. To correct the $x$ coordinates for the nonlinear functional form of the $B_{1}$ gradient, the calculated field profile from a cylindrical coil [Eq. (2)] was used. As a consequence of the nonlinear gradient the voxel size and the spatial resolution increase for longer distances from the coil. For our sample, the resolution over the image decreased by $\sim 50 \%$ from the voxel closest to the voxel furthest from the rf coil. The intensity of an image point is proportional to the number of spins within the voxel volume and thus the larger voxels will have larger intensities. To obtain voxel intensities that encode the spin density, this effect is compensated by normalizing the intensity of each voxel by its length in the $x$ direction. 

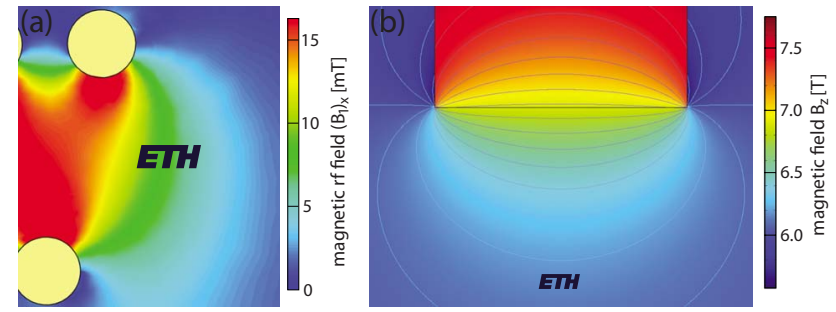

FIG. 4. (Color online) (a) Simulation of the rf field from the coil used in our experiments (Ref. 34). The diameter of the wire, $30 \mu \mathrm{m}$, can be used as a size scale. (b) Simulation of the static magnetic field of the iron gradient source (cylinder diameter $200 \mu \mathrm{m}$ ) magnetized in an external 6.0 T field (Ref. 35). The colors display the field in the $z$ direction and the contours show the lines of constant field amplitude with a step distance of $100 \mathrm{mT}$ and the dashed white line is the central sensitive slice in the sample at 6.176 T.

For the $z$ direction we need not to correct the intensity for the different thicknesses of the slices as explained above for the $x$ reconstruction because the thinner slices are acquired with the higher sensitivity of a higher gradient, which compensates the measured signal for the lower number of spins in the smaller volume.

\section{DISCUSSION}

The spatial resolution of our image is around $1 \mu \mathrm{m}$ and represents a significant improvement over classical microimaging. The obtained resolution is essentially sensitivity limited and further technical improvement will lead to increased resolution, even for samples at room temperature. Possible improvements will be discussed below together with the fundamental limitations and how these influence the image resolution.

Instrumental measures to improve the SNR, and thereby the resolution, include a smaller gradient source which could be placed closer to the sample. The SNR is proportional to the gradient strength (at fixed spatial resolution) which scales inversely proportional to the diameter of the cylindrical source. A gradient-source diameter that matches the size of the object could provide a factor of more than 5 in sensitivity [see Fig. 4(b)] over the setup used in the experiments described here. With a smaller gradient source, the repositioning of the gradient (after flipping it away) is however more critical and the experiment is more susceptible to long term drifts in the relative position of sample and gradient source. Furthermore, the $Q$ factor of the cantilevers could be significantly improved over the value of 3000 used in our experiments and could exceed $40000 .^{21,22}$ As the SNR scales with $\sqrt{Q}$, this could yield another factor of 3 , approximately. Obviously SNR can also be improved by going to lower temperatures. ${ }^{10}$

Beyond the SNR issue, which limits the resolution in the experiments presented, the spatial resolution of our Fourier and Hadamard encoding is limited by the achievable spectral resolution. MRFM experiments operate in the near field, as the sample dimensions and the distance to the coil are much smaller than the rf wavelength and all parts of the sample feel the same rf phase. Therefore, resolving two volume elements in space requires that the resonance frequencies of the two elements must be spectrally resolved. The following condition must be fulfilled: $\Delta x=\frac{\Delta \nu}{2 \pi \gamma G}$ with the gyromagnetic ratio $\gamma$, the gradient strength $G$, and the resolution requested $\Delta \nu$. The linewidth of the sample $\Delta \nu$ is given either by spinspin interactions (e.g., the dipole coupling) or by relaxation effects. For solids, the former usually dominate and because of their deterministic nature, the spectral resolution can be improved by using line-narrowing methods. The limits in the spatial resolution can thus be addressed by two means: either by increasing the gradient strength $G$ or reducing the spectral linewidth $\Delta \nu$ by selective averaging pulse schemes. To discuss this point further we need to treat the $x$ and $z$ directions separately.

In the $z$ direction, encoding (using Hadamard or other schemes) requests that the spectral bandwidth that corresponds to the desired spatial resolution is of the same order or larger than $\Delta \nu$ which, for our sample, amounts to $\sim 30 \mathrm{kHz} .{ }^{23}$ It could be reduced by increasing the gradient in the static field or by using rf schemes that provide homonuclear decoupling. ${ }^{24}$

During $x$ encoding the dipolar coupling is scaled by a factor $-\frac{1}{2}$ by on-resonance rf irradiation. ${ }^{25}$ Magic-echo sequences would allow for efficient homonuclear decoupling and the linewidth under the nutation pulses could potentially be reduced. ${ }^{26,27}$ In our experiment we found a linewidth of $4.5 \mathrm{kHz}$ corresponding to a maximal spatial resolution of 400 $\mathrm{nm}$.

To further increase the resolution in the direction of the $B_{1}$ encoding, the $B_{1}$-gradient strength could be improved. Simply scaling down the size of the rf coil-including the thickness of the wires-does however not increase the gradient, assuming the current density in the wire is left constant. Nonetheless more optimal rf geometries could be imagined, i.e., a Maxwell coil pair or a strip line could replace the single solenoid coil. Of course, the simplest fashion of increasing the gradient would be to send a higher current through the wire, which was limited by the amplifier power in our case.

The achievable resolution thus depends on the spin-spin couplings in the sample. Less rigid samples will be easier to handle because of motional narrowing of the resonance line. The techniques used in this paper could be improved to produce a resolution of below $100 \mathrm{~nm}$ but they are not intended to provide single-spin resolution.

In comparison to scanned-probe imaging in MRFM (Refs. 11-15) the Hadamard/Fourier techniques offer some practical features that make applications simpler and more accessible. The methods use a larger field-gradient sourcetypically of comparable size as the largest diameter of the sample - instead of only the imaging depth, and the larger gradient source does not need to be placed as close to the sample, reducing the problems associated with noncontact friction $^{28}$ and offering possibilities to isolate vacuumsensitive samples from the vacuum of the probe chamber. The reduced SNR, as smaller gradient dimensions reduce the field gradient and the force on the cantilever per voxel, can be approximately compensated by the multiplex advantage of the Hadamard/Fourier schemes employed. With respect to 
the deconvolution procedure needed to reconstruct a spatial image from the raw data, the Fourier/Hadamard method is simple and less prone to artifacts as compared to scanningprobe methods. Furthermore, it offers the possibility to selectively image slices at any chosen $z$ position in the sample, saving acquisition time. An alternative Fourier-encoding method which requires synchronizing the motion of the cantilever with a series of rf pulses was proposed by Marohn and $\mathrm{Kempf}^{29}$ and could offer similar advantages as the method we work with here, but it remains to be demonstrated experimentally.

\section{CONCLUSIONS}

In this study, we present an alternative to the scannedprobe imaging standard in MRFM. The method allows for simultaneous acquisition of signals from the entire sample providing the maximal multiplexing effect. We have experimentally demonstrated a resolution of about $1 \mu \mathrm{m}$. In most solid samples a resolution around $100 \mathrm{~nm}$ should be feasible. The technique is expected to be useful for applications in materials science and is a step toward functional imaging of biologically relevant samples; for example, a single cell. If a selection of the multitude of MRI imaging methods presently applied to living organisms could be applied to living cells, this could open an intriguing new perspective.

\section{ACKNOWLEDGMENTS}

We thank the Schweizerischer Nationalfonds (SNF), the ETH Zurich, and the Kommission für Technologie und Innovation (KTI) for financial support. We thank K. Sankaran for scientific discussions.

\footnotetext{
*beme@ethz.ch

${ }^{1}$ L. Ciobanu, D. A. Seeber, and C. H. Pennington, J. Magn. Reson. 158, 178 (2002).

${ }^{2}$ P. Glover and P. Mansfield, Rep. Prog. Phys. 65, 1489 (2002).

${ }^{3}$ D. E. Demco and B. Blümich, Concepts Magn. Reson. 12, 188 (2000).

${ }^{4}$ D. E. Demco and B. Blümich, Concepts Magn. Reson. 12, 269 (2000).

${ }^{5}$ J. A. Sidles, Phys. Rev. Lett. 68, 1124 (1992).

${ }^{6}$ J. A. Sidles, Appl. Phys. Lett. 58, 2854 (1991).

${ }^{7}$ J. A. Sidles, J. L. Garbini, K. J. Bruland, D. Rugar, O. Zuger, S. Hoen, and C. S. Yannoni, Rev. Mod. Phys. 67, 249 (1995).

${ }^{8}$ D. Rugar, R. Budakian, H. J. Mamin, and B. W. Chui, Nature (London) 430, 329 (2004).

${ }^{9}$ N. Nestle, A. Schaff, and W. S. Veeman, Prog. Nucl. Magn. Reson. Spectrosc. 38, 1 (2001).

${ }^{10}$ J. A. Sidles and D. Rugar, Phys. Rev. Lett. 70, 3506 (1993).

${ }^{11}$ O. Zuger and D. Rugar, Appl. Phys. Lett. 63, 2496 (1993).

${ }^{12}$ O. Zuger, S. T. Hoen, C. S. Yannoni, and D. Rugar, J. Appl. Phys. 79, 1881 (1996)

${ }^{13}$ S. H. Chao, W. H. Dougherty, J. L. Garbini, and J. A. Sidles, Rev. Sci. Instrum. 75, 1175 (2004).

${ }^{14} \mathrm{~S}$. Tsuji, T. Masumizu, and Y. Yoshinari, J. Magn. Reson. 167, 211 (2004).

${ }^{15}$ H. J. Mamin, M. Poggio, C. L. Degen, and D. Rugar, Nat. Nanotechnol. 2, 301 (2007).

${ }^{16}$ D. I. Hoult, J. Magn. Reson. 33, 183 (1979).

${ }^{17}$ D. Canet, Prog. Nucl. Magn. Reson. Spectrosc. 30, 101 (1997).

${ }^{18}$ P. C. Lauterbur, Nature (London) 242, 190 (1973).

${ }^{19}$ A. Kumar, D. Welti, and R. R. Ernst, Naturwiss. 62, 34 (1975).
}

${ }^{20}$ K. W. Eberhardt, C. L. Degen, and B. H. Meier, Phys. Rev. B 76, 180405(R) (2007).

${ }^{21}$ S. Mouaziz, G. Boero, G. Moresi, C. L. Degen, Q. Lin, B. H. Meier, and J. Brugger, Microelectron. Eng. 83, 1306 (2006).

${ }^{22}$ H. J. Mamin and D. Rugar, Appl. Phys. Lett. 79, 3358 (2001).

${ }^{23}$ C. L. Degen, Q. Lin, A. Hunkeler, U. Meier, M. Tomaselli, and B. H. Meier, Phys. Rev. Lett. 94, 207601 (2005).

${ }^{24}$ J. D. Walls, M. Marjanska, F. Castiglione, and A. Pines, Chem. Phys. Lett. 357, 241 (2002).

${ }^{25}$ W. K. Rhim, A. Pines, and J. S. Waugh, Phys. Rev. B 3, 684 (1971).

${ }^{26}$ G. S. Boutis, P. Cappellaro, H. Cho, C. Ramanathan, and D. G. Cory, J. Magn. Reson. 161, 132 (2003).

${ }^{27}$ C. L. Degen, Q. Lin, and B. H. Meier, Phys. Rev. B 74, 104414 (2006).

${ }^{28}$ B. C. Stipe, H. J. Mamin, T. D. Stowe, T. W. Kenny, and D. Rugar, Phys. Rev. Lett. 87, 096801 (2001).

${ }^{29}$ J. G. Kempf and J. A. Marohn, Phys. Rev. Lett. 90, 087601 (2003).

${ }^{30}$ Q. Lin, C. L. Degen, M. Tomaselli, A. Hunkeler, U. Meier, and B. H. Meier, Phys. Rev. Lett. 96, 137604 (2006).

${ }^{31}$ C. L. Degen, U. Meier, Q. Lin, A. Hunkeler, and B. H. Meier, Rev. Sci. Instrum. 77, 043707 (2006).

${ }^{32}$ D. Rugar, O. Zuger, S. Hoen, C. S. Yannon, H. M. Vieth, and R. D. Kendrick, Science 264, 1560 (1994).

${ }^{33}$ A. Schaff and W. S. Veeman, J. Magn. Reson. 126, 200 (1997).

${ }^{34}$ CST, Inc., CST MWS v. 2008.03, CST, Darmstadt, 2008.

${ }^{35}$ COMSOL, Inc., COMSOL Multiphysics v. 3.2, COMSOL, Stockholm, 2005. 